\title{
BCC or not: Sufu keeps it in check
}

Editorial

\section{Wen-Chi Yin, Zhu Juan Li, and Chi-chung Hui}

Basal cell carcinoma (BCC), driven by aberrantly activated HEDGEHOG (HH) pathway, is the most common human malignancy. Current FDA-approved targeted therapy uses Vismodegib to inhibit SMO, a membrane component of the HH pathway. Despite initial impressive tumor regression, the positive clinical response is short-lived in some BCC patients as acquired SMO mutations confer secondary resistance[1]. Clearly, a deeper understanding of the molecular events underlying $\mathrm{BCC}$ tumorigenesis is required to devise effective treatments.

The activity of SMO is repressed by the $\mathrm{HH}$ receptor PTCH1. Upon HH binding, SMO promotes dissociation of GLI transcription factors from the key negative intracellular regulator SUFU, thereby allowing expression of $\mathrm{HH}$ target genes[2]. Mutations in PTCH1, $S M O$, and $S U F U$, believed to unleash GLI activity, are frequently found in BCC. SUFU, like PTCH1, is a major negative regulator of the $\mathrm{HH}$ pathway. We have previously shown that loss of Sufu in mouse keratinocytes promotes Gli2 nuclear localization due to lack of cytoplasmic sequestration, and consequently leads to elevated target gene expression[3]. Surprisingly, unlike Ptch1, inactivation of $S u f u$ alone in the mouse skin does not cause BCC.

To identify the key oncogenic events in BCC formation, we performed microarray coupled with Gene Set Enrichment Analysis on Ptchl and Sufu mutants[4]. The comparative analysis revealed that loss of Ptchl in keratinocytes led to significant enrichment of gene sets involved in TGF- $\beta$ signaling and extracellular matrix remodelling, consistent with the tumorigenic phenotype. In contrast, the majority of gene sets uniquely enriched in Sufu knockout keratinocytes are involved in cell cycle control, suggesting a novel role of Sufu in cell cycle regulation. Intriguingly, unlike Ptchl knockout skin, which showed elevated number of mitotic cells, Sufu knockout skin exhibited normal mitotic count. Furthermore, while DNA damage was found in both mutants, Sufu knockout cells displayed DNA damageinduced $\mathrm{G}_{2} / \mathrm{M}$ checkpoint cell cycle arrest. These results indicate that Ptch1 knockout cells are able to override the checkpoint and continue proliferation with the unstable genome while Sufu knockouts halt, a key feature likely contributing to their differential cancer phenotypes. Arrest at $\mathrm{G}_{2}$ is typically coupled with accumulation of $\mathrm{p} 53$, which activates p21 and 14-3-3 $\sigma$ to sequester mitosis-promoting complex Cyclin-B1/CDK1. Strikingly, p53 protein and p21 transcripts remained low in Sufu mutants despite the arrest. These findings suggest that while both loss of Sufu and Ptch1 result in increased entry into cell cycle and impairment in p53 response to cell cycle-driven DNA damage, Sufu itself may be a positive regulator of cell cycle progression independent of the $\mathrm{p} 53$ checkpoint.

Upregulation of the major $\mathrm{HH}$ pathway effector, Gli2, is a hallmark of BCC and is observed in Ptch1 mouse models. Consistent with our finding that loss of Ptchl leads to genome instability and evasion of cell cycle checkpoints, Pantazi et al. [5] recently demonstrated that overexpression of GLI2 activator $(\mathrm{GLI} 2 \Delta \mathrm{N})$ in human keratinocytes is sufficient to induce chromosomal aberrations. They also found that GLI $2 \Delta \mathrm{N}$ overexpression results in suppression of cell cycle regulators p21 and 14$3-3 \sigma$, and induction of anti-apoptotic mechanisms. These lines of evidence suggest that GLI2 is likely the major mediator of the malignant transformation induced by the loss of $\mathrm{PTCH} 1$ during $\mathrm{BCC}$ tumorigenesis.

In vitro studies demonstrated that $\mathrm{HH}$ signaling can positively regulate cell cycle by promoting the expression of cell cycle regulators (D-type cyclins) and preventing the accumulation of $\mathrm{p} 53$. These are consistent with the active mitosis and evasion of cell cycle arrest observed in Ptch1

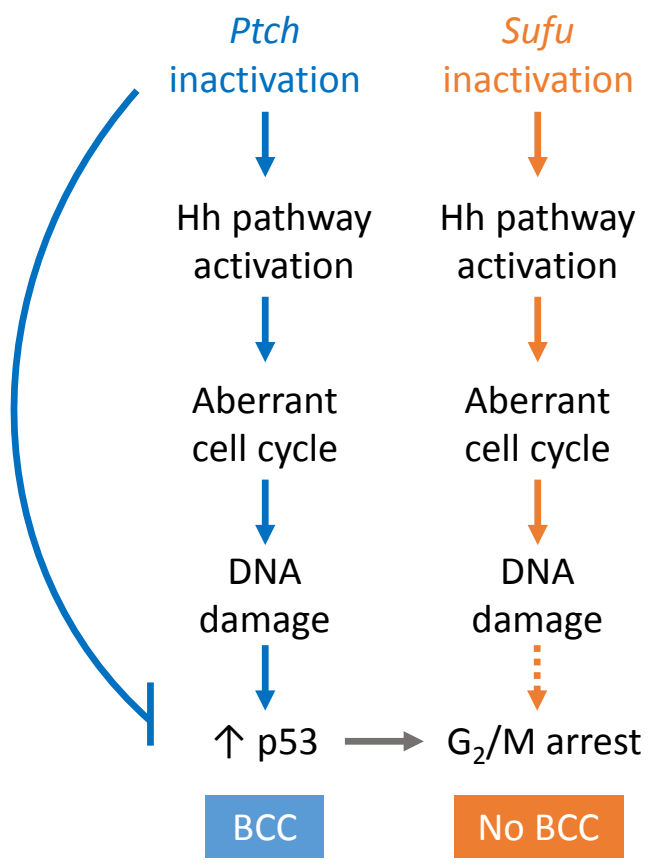

Figure 1: Inactivation of Ptch1 and Sufu lead to distinct cellular events in keratinocytes 
knockout cells. Our findings suggest that Sufu may also regulate cell cycle. However, it remains unclear why and how the loss of this negative $\mathrm{HH}$ pathway regulator causes cell cycle arrest. One possible mechanism is through DNA damage response, which involves the ATM/ATR, CHK1/ CHK2, and CDC25C axis to inactivate the Cyclin-B1/ CDK1 complex, leading to $\mathrm{G}_{2}$ arrest.

Whether Sufu's cell cycle function is Gli-dependent is also unknown. Although ectopic $\mathrm{HH}$ target gene expression was found in both Sufu and Ptch1 mutants, Gli2 protein is significantly reduced in Sufu mutants compared to wildtype, with exclusive nuclear localization. It is possible that a certain threshold of Gli2 activity is required for evasion of cell cycle arrest and tumor surveillance, and that BCC tumorigenesis is stunted in Sufu mutants since the threshold is not achieved.

Double knockout of Sufu and Ptch1 may help determine whether Sufu is required for the rapid cell cycle progression induced by loss of Ptch1. In addition, with the recent advances in BioID mass spectrometry[6], identification of Sufu's interactome in keratinocytes may give mechanistic insights into Sufu's involvement in cell cycle regulation. In conclusion, this comparative study of
Ptchl and Sufu mutant mice advanced our understanding of BCC tumorigenesis. Further investigations elucidating the role of Sufu in the cell cycle are warranted for the reason that if Sufu can also function as a positive regulator of the HH pathway, it may represent a potential target for therapeutic intervention of BCC.

Chi-chung Hui: Developmental and Stem Cell Biology, Hospital for Sick Children, Toronto, Canada.

Correspondence: Chi-chung Hui, email cchui@sickkids.ca

Received: February 10, 2015;

Published: February 20, 2015;

\section{REFERENCES}

1. Atwood SX et al. Cold Spring Harb Perspect Med. 2014; $4: 1-12$.

2. Hui CC et al. Annu Rev Cell Dev Biol. 2011; 27:513-537.

3. Li ZJ et al. Development. 2012; 139:4152-4161.

4. Li ZJ et al. Oncogene. 2014; 33:2674-2680.

5. Pantazi E et al. Cell Death Dis. 2014; 5:e1028.

6. Roux et al. J Cell Bio. 2012; 196:801-810. 